\title{
TRIAGEM DE INDICADORES DE ALTAS HABILIDADES/SUPERDOTAÇÃO: VALIDADE DE CRITÉRIO
}

(D) TATIANA DE CÁSSIA NAKANO'

(D) RICARDO PRIMI"

\author{
Pontifícia Universidade Católica de Campinas (PUC-Campinas), Campinas-SP, Brasil; \\ tatiananakano@hotmail.com \\ "Universidade São Francisco (USF), Campinas-SP, Brasil; rprimi@mac.com
}

\section{RESUMO}

O estudo buscou investigar as evidências de validade de critério de um instrumento intitulado Triagem de Indicadores de Altas Habilidades/Superdotação (AH/S). A escala, respondida pelo professor, avalia o desenvolvimento do estudante em cinco áreas: capacidade intelectual geral, habilidades acadêmicas específicas, liderança, criatividade e talento artístico. A amostra foi composta por 568 participantes: 213 do grupo-controle e 355 do grupo-critério. Os resultados da análise de variância fatorial e do teste $t$ de Student indicaram diferenças de médias significativas entre os grupos, confirmando o tipo de evidência de validade investigada. A regressão logística também foi conduzida visando identificar o quanto a pontuação em cada área específica da escala conseguiria prever a área de identificação do indivíduo com AH/S. 


\section{CLASIFICACIÓN DE INDICADORES DE ALTAS HABILIDADES/ SUPERDOTACIÓN: VALIDEZ DE CRITERIO}

\section{RESUMEN}

El estudio procuró investigar las evidencias de la validez de criterio de un instrumento llamado Identificación de Indicadores de Altas Habilidades/Superdotación. La escala, respondida por el profesor, evalúa el desarrollo del estudiante en cinco áreas: capacidad intelectual general, habilidades específicas para estudios, liderazgo, creatividad y talento artístico. La muestra fue compuesta por 568 participantes: 213 del grupo-control y 355 del grupo-criterio. Los resultados del análisis de varianza factorial y del Test t de Student indicaron diferencias significativas de promedios entre los grupos, confirmando las evidencias investigadas. La regresión logística también fue realizada, teniéndose el objetivo de identificar cuánto la puntuación en cada área específica de la escala conseguía prever el área de identificación del individuo con altas habilidades/superdotación.

PALABRAS CLAVE ALUMNO CON ALTAS HABILIDADES/SUPERDOTACIÓN • VALIDEZ ESTADÍSTICA・ELABORACIÓN DE TEST・EVALUACIÓN PSICOLÓGICA.

\section{GIFTED INDICATORS SCREENING SCALE: CRITERION VALIDITY}

\section{ABSTRACT}

The study aimed to investigate evidence of criterion validity of an instrument named Screening of Indicators of High Skills/Giftedness. The scale, answered by the teacher, evaluates the student's development in five areas: general intellectual ability, specific academic abilities, leadership, creativity and artistic talent. The sample consisted of 568 participants: 213 in the control group and 355 in the criterion group. The results of factorial variance analysis and the Student t-test indicated significant differences of the means between groups, confirming the evidence investigated. Logistic regression analysis was also conducted to identify how much the score in each specific area of the scale could predict the area high skills/giftedness identified for the individual. 


\section{INTRODUÇÃO}

No Brasil, o Ministério da Educação define os alunos com Altas Habilidades/ Superdotação $(\mathrm{AH} / \mathrm{S})$ como aqueles que, além de apresentarem um alto potencial, combinado ou isolado, nas áreas intelectual, acadêmica, de liderança e psicomotricidade, manifestam uma elevada criatividade, e um alto envolvimento com a aprendizagem e com a realização de tarefas de seu interesse (BRASIL, 2010). O presente estudo adota essa definição, que vai ao encontro das acepções mais aceitas internacionalmente para o fenômeno (LI et al., 2009; ROBINSON; CLINKENBEARD, 2008).

Considerando estimativas da Organização Mundial de Saúde (OMS) que indicam que cerca de $3 \%$ a $5 \%$ da população apresenta $\mathrm{AH} / \mathrm{S}$, somente no Brasil deveria haver por volta de 2,5 milhões de alunos identificados (PÉREZ, 2012). No entanto a consulta aos dados do Censo Escolar de 2018 aponta para a subnotificação dos casos, visto que o número de alunos identificados, na ocasião, era de 23 mil estudantes, ou seja, nem $0,00047 \%$ dos 48,5 milhões de estudantes matriculados na educação básica regular, segundo o Instituto Nacional de Estudos e Pesquisas Educacionais Anísio Teixeira (Inep) (BRASIL, 2018). No ensino superior, o Censo realizado pela mesma instituição aponta que esse número é de 1.067 alunos (BRASIL, 2018). A discrepância entre os números do Censo e a estimativa aponta para dificuldades na identificação desses indivíduos.

Ainda que a Lei de Diretrizes e Bases da Educação Nacional (LDBEN) de 1996 (BRASIL, 1996) apresente um artigo que obriga estados, Distrito Federal e municípios a estabelecerem diretrizes e procedimentos para identificação, cadastramento e atendimento, na educação básica e na superior, de alunos com altas habilidades/ superdotação, na prática a situação é bem diferente. Como são as escolas que respondem aos questionários do Censo Escolar, o reduzido número de estudantes identificados sugere que os professores não estão conseguindo constatar o potencial desses alunos (FARIAS; WECHSLER, 2014).

Outra dificuldade enfrentada refere-se ao fato de que, embora as AH/S sejam caracterizadas pela presença de uma habilidade acima da média que pode se manifestar em diferentes aspectos, como área acadêmica, intelectual, criatividade, artes, área psicomotora, habilidades interpessoais e liderança (KAUFMAN; STERNBERG, 2008), na prática, a identificação, quando ocorre, tipicamente se centra na avaliação das habilidades intelectuais e acadêmicas, sendo raramente identificados os demais tipos (JAROSEWICH; PFEIFFER; MORRIS, 2002). A necessidade de mudar tal quadro se faz presente principalmente perante a constatação de que a identificação é o primeiro passo para que o indivíduo nessa condição possa ampliar suas chances de desenvolver ao máximo seu potencial. Evita também que alguma criança ou adolescente seja erroneamente excluído e deixe de receber o atendimento necessário (PFEIFFER; PETSCHER, 2008; OZCAN; KAYA, 2016). 
Como alternativa para a primeira fase de sinalização (ou despiste, também chamada de screening), instrumentos de aplicação rápida e coletiva têm sido utilizados, principalmente no contexto internacional (PFEIFFER; BLEI, 2008). Sua aplicação se ampara no fato de que, por meio desses instrumentos, é possível coletar dados provenientes de um número grande de sujeitos de uma só vez, podendo tal identificação ser realizada por pais, professores, pares e os próprios alunos. Torna-se necessário, entretanto, que tais instrumentos apresentem uma lista abrangente de comportamentos associados ao fenômeno (ALMEIDA; FLEITH; OLIVEIRA, 2013), de forma que possa cobrir diversas áreas em que o potencial superior pode se manifestar.

No Brasil, a maioria dos alunos que são atendidos em programas especiais para superdotados é admitida por meio de indicações feitas pelos seus professores (ALENCAR; FLEITH; CARNEIRO, 2018). Contudo, segundo as autoras, duas dificuldades se fazem presentes: (1) a maioria dos professores não possui informações sobre AH/S e sobre os serviços disponíveis para esses alunos; e (2) a maior parte dos programas não tem psicólogo na equipe de trabalho, de modo que o uso de testes psicológicos que avaliam criatividade e inteligência, por exemplo, não é frequente.

Com a devida formação, o professor pode atuar como o primeiro responsável pela identificação e encaminhamento desses alunos para avaliação, bem como pela implantação de um atendimento adequado nos casos em que o diagnóstico for confirmado (ALMEIDA et al., 2017). Os instrumentos de screening geralmente incluem a avaliação da presença de comportamentos que são "típicos" de estudantes que apresentam $\mathrm{AH} / \mathrm{S}$, fornecendo informações importantes sobre o nível de desenvolvimento do discente avaliado (HERTZOG et al., 2018) e consistindo, assim, em uma ferramenta de observação sistematizada dos estudantes (CLEVELAND, 2017). Esses profissionais são requisitados a identificar aqueles estudantes que se destacam na sala de aula por apresentar um nível elevado de desenvolvimento em alguma área (ALMEIDA et al., 2016).

Essa avaliação é baseada em um grande número de observações de comportamentos em sala de aula acerca dos domínios que compõem a $\mathrm{AH} / \mathrm{S}$, de forma que a informação obtida pode ser utilizada devido principalmente ao seu baixo custo e à redução de tempo, que normalmente são requeridos durante um processo completo de avaliação. Entretanto cabe salientar que as escalas para professores se constituem em ferramentas auxiliares, não tendo como objetivo substituir os demais procedimentos de identificação e sendo reconhecidas como importante preditor do potencial dos alunos (CLEVELAND, 2017).

Diante da riqueza dos dados que podem ser obtidos por essa fonte, um aumento no número de escalas de avaliação utilizadas para a identificação de 
alunos superdotados pôde ser notado principalmente entre os anos de 1960 e 1990, de acordo com um levantamento realizado por Bracken e Brown (2006), o qual indicou a existência de 31 escalas nos Estados Unidos. Uma revisão, feita por Pfeiffer (2002), apontou as cinco escalas de avaliação mais usadas naquele país: The Gifted Evaluation Scale (MCCARNEY; ANDERSON, 1998); Gifted Rating Scales (PFEIFFER; JAROSEWICH, 2003); Gifted and Talented Evaluation Scales (GILLIAM; CARPENTER; CHRISTENSEN, 1996); Scales for Identifying Gifted Students (RYSER; MCCONNELL, 2004); e Scales for Rating the Behavioral Characteristics of Superior Students (RENZULLI et al., 2002). Outros instrumentos também são encontrados na literatura científica: Scale for Rating the Behavioral Characteristics of the Gifted and Talented Students (SAHIN, 2013); Escala de Habilidades Cognitivas/Aprendizagem (ALMEIDA; OLIVEIRA; MELO, 2000); The Universal Multiple Abilities Scales Examiner's Manual (MCCALLUM; BRACKEN, 2012); Escala de Identificación de Talento Académico (ALEGRÍA; LAY; CALDERÓN; CÁRDENAS, 2010); e The Hispanic Bilingual Gifted Screening Instrument (IRBY; LARA-ALECIO, 1996).

No Brasil, são encontradas outras três escalas. A Escala de Identificação de Dotação e Talento (FREITAS; SCHELINI; PÉREZ, 2017) encontra-se na fase de publicação dos primeiros estudos de investigação das qualidades psicométricas (FREITAS; SCHELINI, 2018), assim como a Escala de Identificação de Talentos pelo Professor (SUÁREZ; WECHSLER, 2019). A Escala de Identificação de Características associadas às Altas Habilidades/Superdotação, com foco no autorrelato, também está sendo estudada (ZAIA; NAKANO, 2020; ZAIA; NAKANO; PEIXOTO, 2018). Destaca-se, no entanto, que o país não conta, até o momento da publicação da ferramenta que será apresentada a seguir, com nenhuma escala de professor voltada a essa finalidade e público específicos que já se encontre publicada.

Visando a preencher essa lacuna e possibilitar ao professor o desempenho de papel mais efetivo na identificação e encaminhamento dos alunos que apresentam sinais indicadores de potencial elevado, bem como reduzir a problemática relacionada à falta de instrumentos de triagem adequadamente validados para esse grupo de estudantes, situação que tem-se mostrado uma persistente dificuldade educacional (MIRANDA; ARAÚJO; ALMEIDA, 2013), foi desenvolvido o instrumento denominado Triagem de Indicadores de Altas Habilidades/ Superdotação (TIAH/S). A TIAH/S pretende constituir-se em uma ferramenta para ser usada como recurso auxiliar na indicação dos indivíduos para um processo amplo de avaliação, podendo ser aplicada por qualquer profissional que atua na educação, especialmente docentes.

Pensada para ser utilizada como um instrumento de screening (sondagem) em uma fase inicial de despiste, a TIAH/S é de aplicação rápida e coletiva, sendo que, 
a partir de seus resultados, os docentes e demais profissionais poderão nomear os alunos que apresentem sinais indicativos da presença de um quadro de $\mathrm{AH} / \mathrm{S}$, os quais deverão ser encaminhados para uma avaliação psicológica mais ampla e aprofundada.

Diante do exposto e considerando a importância de que as qualidades psicométricas de um instrumento sejam investigadas ao longo do seu processo de desenvolvimento, o presente estudo visou a apresentar evidências de validade de critério da ferramenta. No caso aqui relatado, tal fonte de evidência de validade foi utilizada a fim de investigar a eficácia com que o instrumento consegue identificar a probabilidade de pertença a um grupo específico.

\section{PARTICIPANTES}

A amostra, de conveniência, foi composta por 568 participantes com idades de seis a 20 anos (média $=12,58$; desvio-padrão $=2,28$; sendo 15 de seis a oito anos, 77 de nove a dez anos, 180 de 11 a 12 anos, 173 de 13 a 14 anos, 81 de 15 a 16 anos, 22 de 17 a 18 anos, dois de 19 a 20 anos e 18 não informaram a idade), sendo 235 do sexo feminino, divididos em dois grupos: o grupo-controle e o grupo-critério.

O grupo-controle foi composto por 213 estudantes de 16 escolas regulares localizadas nas regiões Sudeste (Campinas/SP e Vinhedo/SP), Nordeste (São Luís/MA), Sul (Curitiba/PR) e Centro-Oeste (Brasília/DF), sendo duas escolas particulares, 13 públicas (municipais e estaduais) e um colégio militar, sem diagnóstico de alta habilidade/superdotação.

O segundo grupo, grupo-critério, foi composto por 355 estudantes identificados com altas habilidades/superdotação, participantes de programas de atendimento (Núcleo de Atividades de Altas Habilidades/Superdotação (NAAH/S) ou salas de recursos) localizados na Região Sul do Brasil (Curitiba/PR, Londrina/PR, Rio do Sul/ SC, Itajaí/SC, Joaçaba/SC, Jaraguá do Sul/SC, Tubarão/SC, Chapecó/SC, Joinville/SC, Campos Novos/SC, Lages/SC, Turvo/SC, Antônio Carlos/SC, São José/SC, Dionísio Cerqueira/SC, Canoinhas/SC, Araranguá/SC, Criciúma/SC, Curitibanos/SC, Grão-Pará/SC e Jaraguá do Sul/SC) e na Região Centro-Oeste (Brasília/DF e Samambaia/ DF). Do total, 340 estudantes eram de Santa Catarina; 82, de São Paulo; 99, do Paraná; 42, do Maranhão; e cinco, do Distrito Federal.

\section{INSTRUMENTO}

\section{Triagem de Indicadores de Altas Habilidades/Superdotação (TIAH/S)}

A escala constitui-se em um instrumento de triagem para avaliação das altas habilidades/superdotação composta por 42 itens, devendo ser respondida pelos 
professores, conforme previamente mencionado. O professor avalia o estudante em relação a cinco áreas do desenvolvimento, mais bem detalhadas a seguir:

- Capacidade intelectual geral (Cap_Int): oito itens que se referem a habilidades e competências gerais, as quais envolvem a capacidade de processar informações e resolver problemas, que está relacionada à memória, capacidade verbal, raciocínio e pensamento abstrato. Exemplo de item: "Aprende, com facilidade e rapidez, a maior parte dos conteúdos ensinados”.

- Habilidades acadêmicas específicas (Hab_Acad): nove itens que dizem respeito ao domínio e destaque em alguma disciplina escolar ou área específica de conhecimento, tais como matemática ou linguagem, dentre outras. Exemplo de item: "Apresenta desempenho superior em matemática".

- Liderança (Lid): oito itens que dizem respeito à capacidade de destacar-se dentre os colegas de classe, de liderar e tomar decisões e de influenciar os demais. Exemplo de item: "Destaca-se quando se encontra trabalhando em grupo".

- Criatividade (Criat): oito itens que envolvem formas originais e diferentes de lidar com os problemas e situações, presença de flexibilidade de pensamento, grande número de ideias, imaginação e curiosidade. Exemplo de item: "Apresenta curiosidade bastante aguçada".

- Talento artístico (Tal_Art): nove itens que avaliam interesses e talentos em áreas como música, dança, arte, teatro, desenho, pintura. Exemplo de item: "Domina algum instrumento musical".

O instrumento foi desenvolvido com base na Gifted Rating Scale (PFEIFFER; JAROSEWICH, 2003) e avalia as mesmas áreas, com exceção de motivação. De modo análogo, embasou-se na definição de AH/S adotada pelo Ministério da Educação (BRASIL, 2010), avaliando cinco das áreas propostas (intelectual, acadêmica, liderança, criatividade e talento artístico). Futuramente, almeja-se que outras áreas sejam incorporadas à ferramenta (psicomotricidade, motivação, aspectos socioemocionais), a fim de ampliar sua abrangência.

Durante o processo de avaliação do aluno, o professor deve assinalar com um " $x$ " a alternativa que melhor descreve o aluno que está sendo avaliado. Há quatro opções: abaixo da média se comparado a estudantes da mesma turma (opção em que se pontua 1); nível de habilidade igual a de outros estudantes (opção em que se pontua 2); acima da média apresentada pelos outros estudantes (opção em que se pontua 3); ou então, caso o professor não se sinta apto para sinalizar o que melhor descreve o aluno, ele poderá, na última coluna, marcar um " $x$ " na alternativa "incapaz de julgar" (opção em que se pontua 0). Assim, cada item 
atribui uma pontuação de 0 a 3, e depois são somados os escores das cinco áreas. A métrica final das escalas Cap_Int, Lid e Criat vai de 0 a 24 pontos e das escalas Hab_Acad e Tal_Art, de 0 a 27. É importante ressaltar que notas baixas em uma área podem indicar que o aluno tem um desempenho abaixo do esperado ou que $o$ professor não possuía informações suficientes para julgar o desempenho do aluno naquela área. Por outro lado, a pontuação alta sempre indicará uma percepção do professor de indicadores de AH/S na área elevada. Cada área é seguida por definições para que o professor possa embasar o julgamento feito, visando-se, com isso, a atender à recomendação feita por Pfeiffer e Blei (2008) sobre a necessidade de rigor científico nesse processo de nomeação por professores.

Alguns estudos das qualidades psicométricas do TIAH/S já foram conduzidos, destacando-se a busca por evidências de validade baseadas no conteúdo (NAKANO; CAMPOS; SANTOS, 2016), na relação com variáveis externas (NAKANO et al., 2016; NAKANO et al., 2020) e na estrutura interna (NAKANO; OLIVEIRA, 2019). Durante o processo de normatização da TIAH/S, foram identificados pontos de corte a fim de otimizar a previsão de pertencimento ao grupo-critério, possibilitando a interpretação e o uso da escala com o objetivo de prever se um aluno apresenta características mais próximas daquelas do grupo-critério (identificado com $\mathrm{AH} / \mathrm{S}$ ), considerando as cinco áreas separadamente e a pontuação total do instrumento. Número de pontos iguais ou acima do estabelecido indicaria um alto potencial, variando entre 17 (para a área de liderança) e 22 (para as áreas de capacidade intelectual geral e habilidades acadêmicas específicas).

\section{PROCEDIMENTOS}

Inicialmente o projeto foi aprovado pelo Comitê de Ética em Pesquisa da Pontifícia Universidade Católica de Campinas (PUC-Campinas), sob o número CAAE 21487513.7.0000.5481. A pesquisadora, a princípio, buscou por todos os Núcleos de Atividades de Altas Habilidades/Superdotação listados na página do Ministério da Educação. Para cada um deles, enviou um e-mail explicando a finalidade da pesquisa. É importante ressaltar que a maior parte dos referidos e-mails retornou com uma mensagem indicando que o endereço não mais existia ou estava incorreto.

Por outro lado, uma instituição localizada no estado de Santa Catarina respondeu afirmativamente, colocando-se à disposição para auxiliar na coleta de dados. Por esse motivo, a maior parte da amostra do grupo-critério é proveniente desse estado. A pesquisadora realizou o treinamento da coordenadora da instituição para a aplicação das escalas. Todos os professores e profissionais que atuavam nos polos de atendimento educacional especializado, em um total de 20 cidades, tiveram 
acesso ao link da escala, on-line na plataforma Google Docs. A eles foi solicitado que avaliassem os alunos que orientavam, respondendo a um questionário da escala para cada aluno, indicando ainda a área em que a $\mathrm{AH} / \mathrm{S}$ tinha sido identificada. Ainda em relação ao grupo-critério, a pesquisadora também teve auxílio de duas outras pesquisadoras de sua rede de contatos, as quais realizaram a coleta de dados nos NAAH/S do Distrito Federal e em salas de recursos localizadas no estado do Paraná, seguindo os mesmos procedimentos.

Em relação ao grupo-controle, a coleta de dados foi realizada com o instrumento no formato lápis e papel, em salas de aula regulares de 16 escolas diferentes. Os professores foram convidados a responder o questionário da escala enquanto seus alunos participavam de outra pesquisa, também para identificação das $\mathrm{AH} / \mathrm{S}$, mas a partir de outros instrumentos, a saber, de desempenho ou de autorrelato. Aos professores solicitou-se que respondessem a quantos questionários da escala pudessem, selecionando os alunos que não apresentavam, na sua opinião, destaque em nenhuma área quando comparados com os demais estudantes da sala de aula.

\section{PLANO DE ANÁLISE DE DADOS}

A princípio foi criada uma planilha com os dados do grupo-controle e do grupo experimental (critério), contendo a resposta dos professores para cada um dos 42 itens da escala. Em seguida, a pontuação bruta em cada uma das áreas avaliadas foi estimada. A precisão por consistência interna (alfa de Cronbach) de cada área foi: capacidade intelectual geral $\alpha=0,95$, habilidades acadêmicas específicas $\alpha=$ 0,90 , liderança $\alpha=0,93$, criatividade $\alpha=0,89$ e talento artístico $\alpha=0,91$. A precisão do escore total foi $\alpha=0,97$.

A fim de verificar se a escala conseguiria diferenciar o desempenho entre os grupos controle e critério, inicialmente foram obtidas estatísticas descritivas sobre os escores de cada grupo. Posteriormente foi utilizada a análise de variância fatorial com três fatores (área: 5 níveis; grupo: 2; e sexo: 2), testando-se principalmente a diferença entre as médias das pontuações dos grupos e sua interação com áreas avaliadas e sexo. Finalmente foi aplicada uma regressão logística verificando-se a capacidade das escalas em predizer a pertença ao grupo-critério. A partir dessa análise foram identificados pontos de corte otimizados para auxiliar na interpretação referenciada ao critério das escalas e da pontuação total. Todas as análises foram feitas no R (R CORE TEAM, 2018) e no jamovi (THE JAMOVI PROJECT, 2019; SELKER; LOVE; DROPMANN, 2019). Para determinação dos pontos de corte, utilizou-se o pacote OptimalCutpoints (LÓPEZ-RATÓN et al., 2014; LÓPEZ-RATÓN; RODRÍGUEZ-ÁLVAREZ, 2019). 


\section{RESULTADOS}

Os resultados da estatística descritiva aplicada aos dados são apresentados na Tabela 1. Revelaram que, para os escores, em todas as áreas avaliadas, o grupo-critério apresentou médias mais altas que aquelas obtidas pelo grupo-controle. Também é possível notar um desempenho que parece ser mais homogêneo no grupo-critério, considerando que os desvios-padrão desse grupo se mostraram menores quando comparados com os apresentados pelo outro grupo.

TABELA 1 - Média e desvio-padrão dos escores por área segundo grupo e sexo

\begin{tabular}{l|c|c|c|c}
\hline ÁREA & GRUPO/SEXO & $\mathbf{N}$ & MÉDIA & DESVIO-PADRÃO \\
\hline \multirow{4}{*}{ Capacidade intelectual geral } & Controle & 213 & 18,11 & 6,10 \\
\cline { 2 - 5 } & Critério & 355 & 22,37 & 3,32 \\
\cline { 2 - 5 } & Feminino & 235 & 20,67 & 5,01 \\
\cline { 2 - 5 } & Masculino & 333 & 20,82 & 5,01 \\
\hline \multirow{4}{*}{ Habilidades acadêmicas específicas } & Controle & 213 & 17,89 & 6,95 \\
\cline { 2 - 5 } & Critério & 355 & 23,53 & 4,69 \\
\cline { 2 - 5 } & Feminino & 235 & 21,35 & 6,14 \\
\cline { 2 - 5 } & Masculino & 333 & 21,38 & 6,41 \\
\hline \multirow{4}{*}{ Tiderança } & Controle & 213 & 15,86 & 6,24 \\
\cline { 2 - 5 } & Critério & 355 & 20,55 & 4,10 \\
\cline { 2 - 5 } & Feminino & 235 & 18,84 & 7,84 \\
\cline { 2 - 5 } & Masculino & 333 & 18,74 & 7,46 \\
\hline \multirow{4}{*}{ Criatividade } & Controle & 213 & 16,17 & 5,88 \\
\cline { 2 - 5 } & Critério & 355 & 21,54 & 3,06 \\
\cline { 2 - 5 } & Feminino & 235 & 19,34 & 4,90 \\
\cline { 2 - 5 } & Masculino & 333 & 19,62 & 7,15 \\
\hline & Controle & 213 & 12,58 & 7,98 \\
\cline { 2 - 5 } & Critério & 355 & 19,91 & 5,84 \\
\cline { 2 - 5 } & Feminino & 235 & 17,09 & 7,84 \\
\hline
\end{tabular}

Fonte: Dados da Pesquisa (elaboração própria).

Em seguida, a fim de verificar se as diferenças encontradas entre os resultados dos grupos eram estatisticamente significantes, efetuou-se uma análise de variância fatorial com três fatores: (área: 5 níveis; grupo: 2; e sexo: 2) sendo área um fator de medidas repetidas. A variável analisada foi o escore (a pontuação) de cada uma das cinco áreas, padronizada em 0 a 1 indicando a proporção da pontuação máxima possível. Testou-se o efeito dos três fatores nas pontuações e as interações entre essas variáveis. Os resultados são apresentados na Tabela 2. 
TABELA 2 - Resultado da análise de variância fatorial

\begin{tabular}{l|c|c|c|c|c|c}
\hline EFEITOS INTRAGRUPOS & SQ & GL & SQM & F & $P$ & $\eta^{2}$ \\
\hline tiahs & 17,044 & 4 & 4,261 & 234,092 & $<0,001$ & 0,293 \\
\hline tiahs $\times$ Grupo & 0,731 & 4 & 0,183 & 10,042 & $<0,001$ & 0,017 \\
\hline tiahs $\times$ Sexo & 0,028 & 4 & 0,007 & 0,390 & 0,816 & 0,001 \\
\hline tiahs $\times$ Grupo $\times$ Sexo & 0,027 & 4 & 0,007 & 0,364 & 0,834 & 0,001 \\
\hline Resíduo & 41,065 & 2256 & 0,018 & & & \\
\hline
\end{tabular}

\begin{tabular}{l|c|c|c|c|c|c}
\hline EFEITOS ENTRE GRUPOS & SQ & GL & SQM & F & P & $\eta_{p}^{2}$ \\
\hline Grupo & 29,470 & 1 & 29,470 & 202,940 & $<0,001$ & 0,265 \\
\hline Sexo & 0,409 & 1 & 0,409 & 2,820 & 0,094 & 0,005 \\
\hline Grupo x Sexo & 0,751 & 1 & 0,751 & 5,170 & 0,023 & 0,009 \\
\hline Resíduo & 81,902 & 564 & 0,145 & & & \\
\hline
\end{tabular}

Fonte: Dados da pesquisa (elaboração própria).

Legenda: $\mathrm{SQ}$ = soma dos quadrados; $\mathrm{gl}$ = graus de liberdade; $\mathrm{SQM}$ = soma dos quadrados médios; $F=$ valor da estatística do teste; $p=$ nível de significância descritivo; $\eta_{p}^{2}=$ eta ao quadrado.

Avaliando em primeiro lugar as comparações das médias intragrupos (fator de medidas repetidas), notou-se que há uma diferença da alta magnitude entre as áreas (tiahs, $\eta_{p}^{2}=0,29$ ). Em geral, as áreas de capacidade intelectual são bem pontuadas, seguidas de habilidades acadêmicas, criatividade e liderança e, por último, talento artístico. Os escores em talento artístico são bem menores do que os escores nas outras áreas. Isso pode indicar que os itens dessa escala se referem a características mais incomuns relativamente às outras áreas. Observou-se uma interação significativa entre áreas e grupo (tiahs $*$ Grupo, $\eta^{2}{ }_{p}=0,017$ ) devido ao fato de que as diferenças entre grupos (critério e controle) são maiores em talento artístico do que nas outras áreas. Não houve interação entre área e sexo, nem entre área, grupo e sexo.

Em seguida, analisando as diferenças entre os estudantes do grupo-critério, pôde-se verificar que eles apresentaram pontuações significativamente mais altas que os do grupo-controle em todas as áreas, de modo a indicar a validade de critério da escala (Grupo $\left.\eta^{2}{ }_{p}=0,26\right)$. Observou-se uma interação significativa, mas de baixa magnitude, entre grupo e sexo (Grupo $*$ Sexo, $\eta^{2}{ }_{p}=0,006$ ), indicando que a diferença de médias entre grupo-controle e critério é maior para meninas do que para meninos. A Figura 1 apresenta as diferenças entre as variáveis grupo e área e a Figura 2, entre sexo e grupo. 
FIGURA 1 - Frequências e médias dos escores padronizados (proporção da pontuação máxima) nas cinco áreas: capacidade intelectual (CI), habilidades acadêmicas (HA), talento artístico (TA), liderança (LI) e criatividade (CR), segundo o grupo (controle e critério)

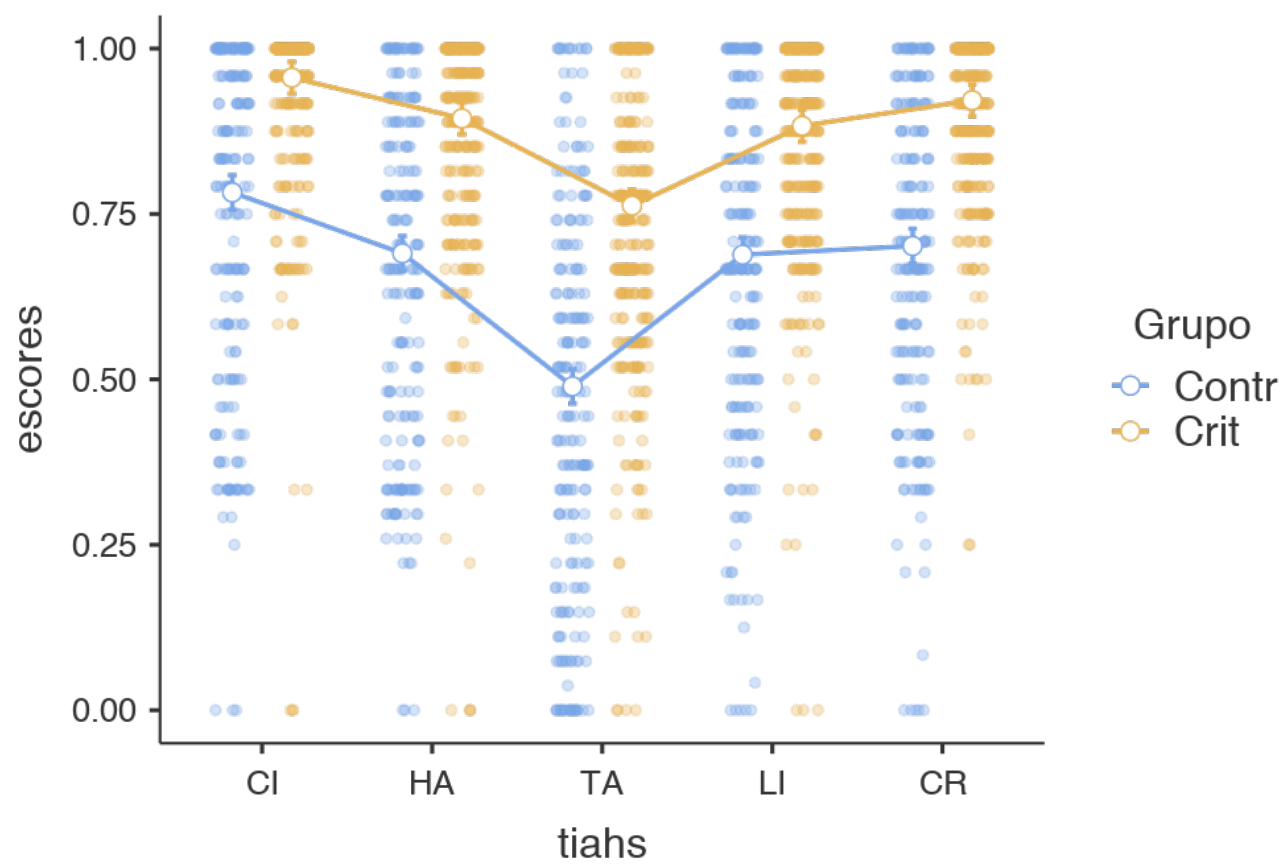

Fonte: Dados da pesquisa (elaboração própria)

FIGURA 2 - Frequência e média dos escores padronizados (proporção da pontuação máxima) do total das cinco áreas, segundo grupo e sexo

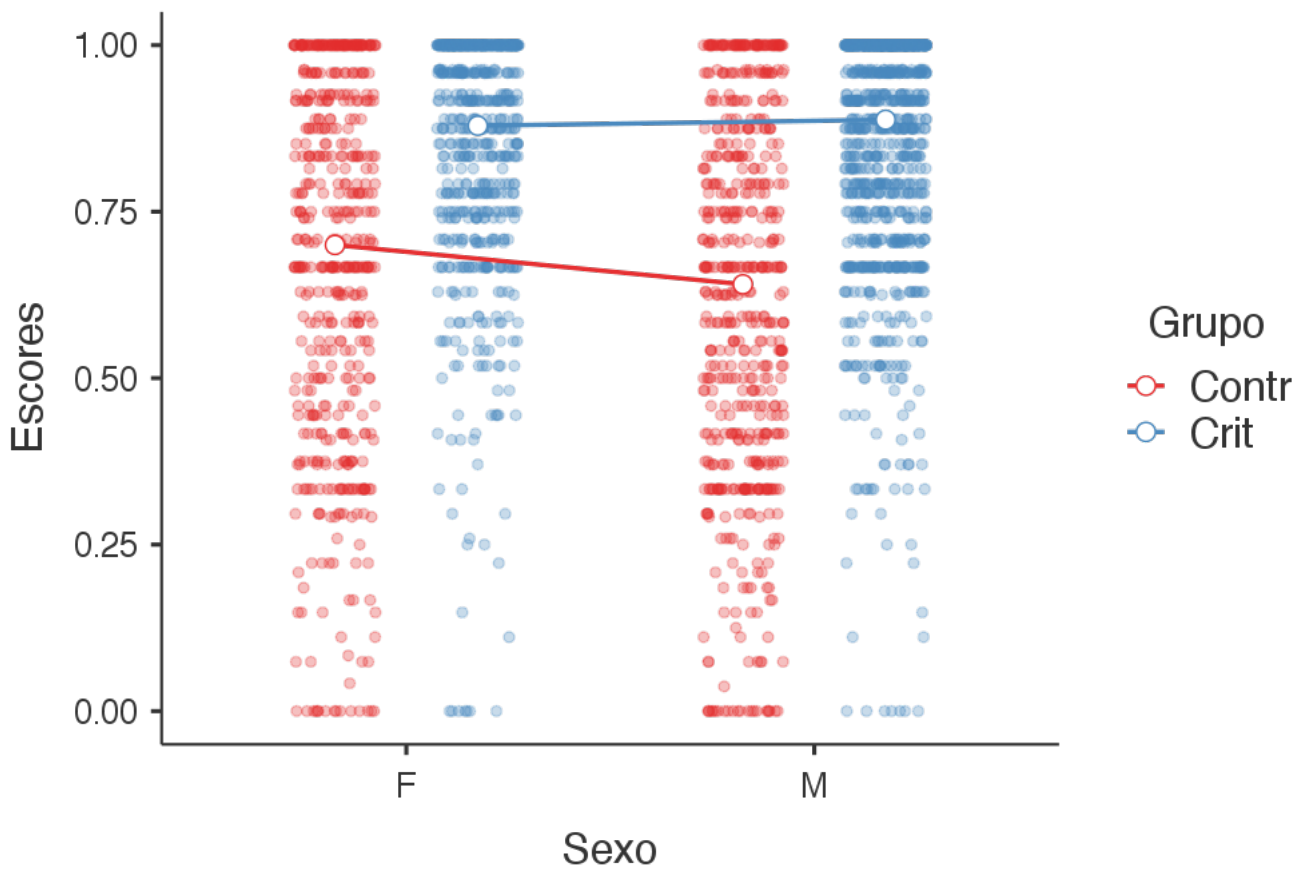

Fonte: Dados da pesquisa (elaboração própria) 
Em seguida, outra análise foi conduzida, considerando a área em que o participante do grupo-critério foi identificado. Para critérios de classificação, um agrupamento das áreas foi realizado. Por exemplo, na área acadêmica, agruparam-se todas as classificações que foram preenchidas pelos professores como de alto desempenho em áreas acadêmicas específicas: matemática, física, literatura, biologia, português, ciências, história, exatas e ciências da natureza. Agruparam-se os participantes que foram classificados pelos professores com esse termo específico (produtivo-criativa), bem como ocorreu com participantes que receberam outras classificações, como área artística, artes e canto. Na área intelectual foram considerados os classificados com esse termo específico, assim como em raciocínio lógico. Por fim, na área denominada tecnologia, foram inseridos aqueles classificados como de alto desempenho em robótica e informática. A distribuição da frequência e porcentagem de cada área é apresentada na Tabela 3, a seguir.

TABELA 3 - Distribuição do grupo-critério conforme área de identificação das AH/S

\begin{tabular}{|c|c|c|}
\hline ÁREA DE IDENTIFICAÇÃO DA AH/S & FREQUÊNCIA & PORCENTAGEM \\
\hline Acadêmica & 152 & 45,5 \\
\hline Produtivo criativa & 60 & 18 \\
\hline Intelectual geral & 12 & 3,6 \\
\hline Tecnologia & 26 & 7,8 \\
\hline Linguagem, códigos e suas tecnologias & 20 & 6 \\
\hline Linguagem & 20 & 6 \\
\hline Interpessoal e intrapessoal & 3 & 0,9 \\
\hline Esporte & 18 & 5,4 \\
\hline Corporal cinestésica & 1 & 0,3 \\
\hline Astronomia & 1 & 0,3 \\
\hline Liderança & 2 & 0,6 \\
\hline Em investigação & 19 & 5,7 \\
\hline Total & 332 & 100 \\
\hline
\end{tabular}

Fonte: Dados da pesquisa (elaboração própria).

É importante destacar que 18 estudantes $(0,05 \%)$ foram apontados como indivíduos em que a identificação das $\mathrm{AH} / \mathrm{S}$ foi feita em mais de uma área: acadêmica e linguagem $(\mathrm{n}=1)$; acadêmica e produtivo-criativa $(\mathrm{n}=4)$; acadêmica e tecnologia $(\mathrm{n}=7)$; intelectual e acadêmica $(\mathrm{n}=2)$; intelectual, produtivo-criativa e acadêmica $(\mathrm{n}=1)$; linguagem e interpessoal $(\mathrm{n}=1)$; linguagem e produtivo-criativa $(\mathrm{n}=1)$; e produtivo-criativa, linguagem e acadêmica $(\mathrm{n}=1)$. Outros 19 participantes ainda estavam em fase de identificação da área (0,05\%). Optou-se por não incluir esses indivíduos na análise posterior, de regressão linear, cujo objetivo era identificar o quanto a pontuação em cada área específica da escala conseguiria prever a área de identificação do indivíduo com AH/S. 
Inicialmente, a correlação de Pearson foi estimada entre as áreas da escala. Os resultados, apresentados na Tabela 4, indicaram correlações diferentes de zero a partir dos testes de significância estatística aplicados entre todas as medidas, oscilando entre $r=0,202$ e $r=0,852$.

TABELA 4 - Coeficientes de correlação de Pearson entre as áreas avaliadas pela escala

\begin{tabular}{l|c|c|c|c}
\hline & HAB_ACAD & LID & CRIAT & TAL_ART \\
\hline Cap_Int & $0,752^{* *}$ & $0,715^{* *}$ & $0,587^{* *}$ & $0,202^{* *}$ \\
\hline Hab_Acad & & $0,718^{* *}$ & $0,560^{* *}$ & $0,312^{* *}$ \\
\hline Lid & & & $0,685^{* *}$ & $0,370^{* *}$ \\
\hline Criat & & & & $0,593^{* *}$ \\
\hline Tal_Art & & & & \\
\hline
\end{tabular}

Fonte: Dados da pesquisa (elaboração própria).

Legenda: Cap_Int = capacidade intelectual geral; Hab_acad = habilidades acadêmicas específicas; Lid = liderança; Criat = criatividade; Tal_Art = talento artístico. ${ }^{*} \mathrm{p} \leq 0,01 ;{ }^{* *} \mathrm{p} \leq 0,001$.

A análise de regressão logística foi conduzida visando a identificar o quanto a pontuação em cada área específica da escala conseguia prever se o estudante fazia parte do grupo-critério. Os resultados indicaram um efeito estatisticamente significativo e único para as áreas talento artístico e criatividade. No entanto isso não quer dizer que as outras áreas tenham contribuição nula para a previsão. Significa que, como as outras áreas têm uma alta correlação entre si, talvez tenham informação redundante umas com as outras e que, apesar de contribuírem igualmente para o escore geral, não têm contribuição única independente entre si. As áreas de talento artístico e criatividade, por outro lado, para além do fator geral, têm uma contribuição específica. Essa análise também permitiu o estabelecimento do número de pontos de corte para criação de normas de interpretação da escala referenciadas ao critério, isto é, definir qual pontuação poderia indicar que o aluno mais se assemelha, na visão do professor, aos alunos identificados com AH/S. 
TABELA 5 - Resultados da regressão logística predizendo pertença ao grupo-critério em função dos escores nas cinco áreas

\begin{tabular}{l|c|c|c|c|c|c|c}
\hline \multirow{2}{*}{ PREDITOR } & \multirow{2}{*}{ COEFICIENTE } & \multicolumn{2}{|c|}{$\begin{array}{c}\text { INTERVALO DE } \\
\text { CONFIANCA (95\%) }\end{array}$} & \multirow{2}{*}{ EP } & Z & P & $\begin{array}{c}\text { ODDS } \\
\text { RATIO }\end{array}$ \\
\cline { 3 - 5 } & & INFERIOR & SUPERIOR & & & & \\
\hline Intercept & $-4,7$ & $-5,82$ & $-3,59$ & 0,569 & $-8,271$ & $<, 001$ & 0,00903 \\
\hline Cap_Int & 0,75 & $-1,14$ & 2,64 & 0,964 & 0,778 & 0,437 & 2,11752 \\
\hline Hab_Acad & 0,844 & $-0,84$ & 2,53 & 0,859 & 0,983 & 0,326 & 2,32663 \\
\hline Tal_Art & 2,114 & 1,14 & 3,08 & 0,494 & 4,281 & $<, 001$ & 8,28337 \\
\hline Lid & $-0,563$ & $-2,14$ & 1,01 & 0,805 & $-0,7$ & 0,484 & 0,56952 \\
\hline Criat & 3,779 & 1,76 & 5,79 & 1,025 & 3,686 & $<, 001$ & 43,78356 \\
\hline
\end{tabular}

Fonte: Dados da pesquisa (elaboração própria).

Legenda: ep = erro padrão; $Z$ = razão entre o coeficiente e o erro padrão; gl = graus de liberdade; Sig.= nível de significância descritivo; Cap_Int = capacidade intelectual geral; Hab_Acad = habilidade acadêmica específica; Tal_art = talento artístico; Lid = liderança; Criat = criatividade .

A análise foi complementada pela aplicação do teste t de Student para cada área da TIAH/S, visando a identificar se a diferença entre os grupos era estatisticamente significativa. Os resultados são apresentados na Tabela 6.

TABELA 6 - Teste $t$ de Student por área e total da escala

\begin{tabular}{l|c|c|c}
\hline ÁREA & T & GL & SIG. (BILATERAL) \\
\hline Capacidade intelectual geral & $-10,759$ & 566 & 0,0001 \\
\hline Habilidades acadêmicas & $-11,522$ & 566 & 0,0001 \\
\hline Liderança & $-10,774$ & 566 & 0,0001 \\
\hline Criatividade & $-14,257$ & 566 & 0,0001 \\
\hline Talento artístico & $-12,533$ & 566 & 0,0001 \\
\hline
\end{tabular}

Fonte: Dados da pesquisa (elaboração própria).

Nota-se que houve uma predição global significativa $\left(X^{2}=185, g l=5, p<001\right)$ com uma sensibilidade de 74,6\%, especificidade de 73,2\% e acurácia geral de $74,1 \%$. As áreas com maior contribuição única significativa foram talento artístico e criatividade, podendo-se dizer que, para além das outras áreas, essas especificamente adicionam informação única à previsão das altas habilidades. Os resultados do teste t também indicaram que as médias em todas as áreas foram significativamente diferentes entre o grupo-controle e o grupo-critério, a favor do segundo, tal como esperado.

Dada a capacidade preditiva das escalas, buscou-se definir os pontos de corte a fim de otimizar a previsão de pertença ao grupo-critério. Esses pontos de corte podem ser usados como informação normativa referenciada ao critério, possibilitando 
a interpretação e o uso da escala, visando a prever se um aluno tem características mais próximas daquelas do grupo-critério aqui estudado. Empregou-se o pacote OptimalCutpoints e o critério baseado no índice de Youden, que maximiza a sensibilidade (proporção de estudantes do grupo-critério identificados corretamente) e a especificidade (proporção do grupo-controle identificados corretamente) (LÓPEZ-RATÓN et al., 2014). Na Tabela 7 são apresentados os números de pontos de corte e os valores da sensibilidade e especificidade associados.

TABELA 7 - Pontos de corte e valores de sensibilidade e especificidade associados

\begin{tabular}{l|c|c|c}
\hline ESCALA & PONTO DE CORTE & SENSIBILIDADE & ESPECIFICIDADE \\
\hline Cap_Int & 22 & 0,80 & 0,58 \\
\hline Hab_Acad & 22 & 0,75 & 0,64 \\
\hline Lid & 17 & 0,86 & 0,53 \\
\hline Criat & 19 & 0,84 & 0,62 \\
\hline Tal_Art & 17 & 0,77 & 0,68 \\
\hline
\end{tabular}

Fonte: Dados da pesquisa (elaboração própria)

Legenda: Cap_Int = capacidade intelectual geral; Hab_Acad = habilidade acadêmica específica; Tal_art = talento artístico; Lid = liderança; Criat = criatividade.

\section{DISCUSSÃO}

Baseando-se no fato ressaltado pela literatura de que a identificação das $\mathrm{AH} / \mathrm{S}$ pode começar na sala de aula a partir da percepção do professor, o processo de construção de uma ferramenta de triagem (screening) de sinais indicativos de AH/S iniciou-se a partir do pressuposto de ampla utilização por profissionais não psicólogos, como primeiro passo na identificação do fenômeno. A prática de triagem dos potenciais alunos pelos professores é comum em muitos países, de modo que os estudantes devem ser encaminhados pelos docentes para que possam ter acesso a uma avaliação (SILVERMAN, 2018). Uma série de estudos já foi conduzida com o instrumental, sendo que a busca por evidências de validade de critério, cujos resultados foram aqui apresentados, confirmaram a capacidade da TIAH/S de discriminar indivíduos que apresentam sinais indicativos daqueles que não os apresentam.

A ferramenta se mostrou eficiente para identificar potenciais nas cinco áreas avaliadas, mostrando-se mais promissora em relação às áreas de talento artístico e criatividade. No estudo, professores apresentaram maior facilidade em identificar as $\mathrm{AH} / \mathrm{S}$ nessas áreas, de modo que três possíveis hipóteses emergiram. A primeira envolveria a possibilidade de que determinada área apresentara, no instrumento, definição mais clara para os participantes, de modo que seus indicadores puderam ser percebidos de forma mais direta pelos professores. É importante destacar que, 
indiferente à área em que a $\mathrm{AH} / \mathrm{S}$ for identificada, características como curiosidade, originalidade e flexibilidade de pensamento, questionamento, impulsividade, abertura a experiências, busca por métodos criativos para resolver problemas e pensamento divergente, que são tipicamente descritoras de indivíduos criativos, fazem-se presentes nos indivíduos superdotados (AL-HROUB; KHOURY, 2018; ALMEIDA; CAPELLINI, 2005; WILLIAMS, 2017). Isso pode ter facilitado a identificação, pelos professores, nas duas áreas citadas.

A segunda hipótese se baseia na distribuição das áreas de identificação da amostra que compõe o grupo-critério. Os estudantes assinalados na área acadêmica se mostraram mais frequentes ( $45,5 \%$ da amostra), seguidos daqueles apontados na área produtivo-criativa (18\%). Se considerarmos que, historicamente, e de forma mais comum, a superdotação tem sido associada a um alto desempenho em inteligência, sendo comum a identificação com base apenas na avaliação desse construto e do desempenho acadêmico (MENDONÇA; RODRIGUES; CAPELLINI, 2015; PFEIFFER; PETSCHER, 2008; RENZULLI, 2008), a predominância desse grupo na amostra analisada pode ser compreendida. Por outro lado, o fato da superdotação produtivo-criativa ter aparecido em segundo lugar, em termos de ocorrência, pode ter exercido alguma influência no julgamento dos professores, os quais tiveram mais facilidade em identificar indicadores nessas áreas, especialmente no caso dos docentes que se encontram atuando nos NAAH/S e salas de recursos, diretamente no atendimento a esses alunos.

Uma possibilidade que não pode ser descartada envolve a potencial presença de um viés interpretativo do fenômeno por parte desses profissionais, que podem apresentar estereótipos de que os superdotados são marcadamente criativos. Outro argumento que nos ajuda a compreender tais resultados baseia-se na constatação de que estudos voltados à investigação da compreensão de professores sobre $\mathrm{AH} / \mathrm{S}$ têm indicado que esses profissionais relatam conhecimento superficial sobre o assunto e consequente dificuldade em lidar com esses alunos (KAUFMAN; ANASTASIOU; MAAG, 2017; MAIA-PINTO; FLEITH, 2002); formação insuficiente durante a graduação ou formação continuada (GONÇALVES, 2017; JOHNSON; SEMMELROTH, 2014; OLIVEIRA; AMORIM; PIZZI, 2018); e busca de orientações por iniciativa própria (VEIGA; GRANDE; GROCHOSKI, 2013). Assim, a presença de mitos associados ao fenômeno pode ter exercido influência direta no julgamento realizado pelos profissionais.

Nesse sentido, considerando que os professores responderam a TIAH/S sem receber nenhuma orientação sobre o fenômeno das $\mathrm{AH} / \mathrm{S}$ e que somente parte da amostra (dos professores que atuavam em serviços especializados de atendimento a esses estudantes) conhecia de forma aprofundada os sinais indicativos, o oferecimento de uma palestra de orientação ou material para os professores da escola 
regular poderia ter sido uma estratégia adotada pelos pesquisadores como forma de tentar controlar esse possível viés. Além disso, ressalta-se que a avaliação das AH/S constitui uma tarefa desafiadora dados alguns aspectos práticos, tais como o inevitável viés de qualquer avaliação subjetiva e as limitações dos atuais testes psicométricos (GUIMARÃES, 2007).

Sinais indicativos da presença de $\mathrm{AH} / \mathrm{S}$ - tais como a ocorrência de algo que o aluno consistentemente faz bem, melhor do que a grande maioria dos pares de idade, facilidade em aprender, boas ideias, comentários interessantes e oportunos, observação detalhada e produção concreta em alguma tarefa (GUENTHER, 2012) - são elementos avaliados na TIAH/S, por meio de itens que se mostraram adequados e seguros do ponto de vista psicométrico. Somados aos resultados dos estudos anteriormente conduzidos com a TIAH/S, os achados atuais permitem verificar que a ferramenta apresenta evidências positivas de validade (NAKANO; CAMPOS; SANTOS, 2016; NAKANO et al., 2016; NAKANO; OLIVEIRA, 2019) baseadas em diferentes fontes, bem como precisão para uso na identificação de sinais indicadores de AH/S (NAKANO; OLIVEIRA, 2019), podendo ser utilizada com segurança por professores como uma ferramenta de triagem e posterior encaminhamento dos casos para uma avaliação psicológica mais aprofundada.

Por fim, convém destacar que a triagem de AH/S no Brasil se mostra uma área promissora, mas que ainda precisa de investimentos. Embora as primeiras políticas públicas voltadas ao atendimento desses alunos datem de 1929 (PEDRO et al., 2016), o que se vê, na prática, é que o Brasil ainda não avançou muito na oferta de atendimento educacional especializado para essa população (DELOU, 2012). O tema continua envolvendo preconceito, desconhecimento e mitos que impedem seu desenvolvimento (VIRGOLIM; KONKIEWITZ, 2014). Uma série de questionamentos relacionados à identificação (variáveis que deveriam ser consideradas, motivos para identificação, métodos e instrumentos a serem utilizados) também merece atenção (ALMEIDA et al., 2016). Especialmente em relação aos instrumentos, faz-se necessário o desenvolvimento de ferramentas que avaliem outras áreas além da inteligência (MENDONÇA; RODRIGUES; CAPELLINI, 2015), de modo a possibilitar a identificação de diferentes perfis. Do mesmo modo, dispositivos que apresentem evidências de validade para esse propósito específico se fazem necessários, dadas as particularidades dessa população (altos níveis de habilidades e singularidades), bem como o fato de que, comumente, indivíduos que apresentam tal quadro alcançam pontuações que vão além dos limites e tabelas normativas dos testes para a população geral (ALMEIDA et al., 2016).

Nesse sentido, Nakano e Campos (2019) destacam uma série de desafios que ainda precisam ser superados na temática e que devem marcar a agenda das $\mathrm{AH} / \mathrm{S}$ : (1) reconhecimento das amplas e diversificadas possibilidades de manifestação do 
fenômeno, (2) melhor definição dos critérios de identificação, (3) desenvolvimento e adaptação dos instrumentos de avaliação, (4) necessidade de que a identificação seja realizada o mais precocemente possível, (5) melhor preparo e formação dos professores e (6) ampliação do oferecimento de programas de atendimento. Reforçam também que a temática das $\mathrm{AH} / \mathrm{S}$ ainda tem se mostrado complexa, principalmente pelas dificuldades relacionadas à sua definição, avaliação e realização de diagnóstico correto. Tais aspectos trazem importantes consequências ao atendimento especializado, seja por conta de ausência dos serviços (ainda que previstos em lei), seja por desconhecimento dos procedimentos por parte de professores e psicólogos.

Dessa forma, o investimento em formação e pesquisa para os profissionais que atuam na área pode gerar contribuições importantes para o processo de identificação e diagnóstico dos indivíduos que apresentam altas habilidades/superdotação, especialmente se considerarmos que tais profissionais vêm sendo desafiados, na sua prática diária, a fazer predições sobre o potencial desses indivíduos ou sua futura performance (NAKANO; CAMPOS, 2019).

\section{CONSIDERAÇÕES FINAIS}

Apesar de a ferramenta ter sido construída com base na definição adotada pelas políticas públicas brasileiras (BRASIL, 2010), no momento de sua operacionalização, somente algumas áreas em que esse potencial superior pode se manifestar foram englobadas: capacidade intelectual geral, habilidades acadêmicas específicas, liderança, criatividade e talento artístico. Outras áreas, como psicomotricidade e motivação, presentes na definição adotada no país, além de outras possibilidades em que o potencial superior pode se manifestar, não foram incorporadas ao instrumento aqui apresentado. Essa característica deve ser interpretada como uma das limitações da ferramenta. A construção de itens para avaliar tais áreas já está sendo pensada, juntamente com itens relacionados a aspectos socioemocionais.

Como pontos fortes a serem ressaltados, destaca-se o número elevado de indivíduos que compuseram o grupo-critério. Sabendo da dificuldade de identificar esses estudantes, bem como do número limitado de locais em que o atendimento a esse público é realizado, a parceria com um centro estadual possibilitou que uma grande amplitude de perfis e áreas de identificação pudesse compor a amostra, de modo a fomentar uma ampla variabilidade de comportamentos cobertos pelas áreas avaliadas pela escala e, assim, confirmar as evidências de validade do instrumento nas cinco áreas que compõem o instrumento. No entanto convém chamar a atenção para o fato de que a maior parte do grupo-critério pertence a uma única região do Brasil, enquanto o grupo-controle é composto por participantes das 
outras regiões. Tal condição pode ter exercido influência nos resultados, de maneira que vieses nos resultados causados por essas diferenças regionais não podem ser desconsiderados. Entretanto, se levarmos em conta que já foram apresentados estudos anteriores comprovando as qualidades psicométricas do instrumento em amostras diversificadas, poderemos assumir que este estudo apresenta evidências robustas de validade e precisão. Estudos futuros poderão investigar, de forma mais aprofundada, a influência das diferenças regionais.

Seus resultados positivos deverão ser somados aos que já foram anteriormente obtidos com a escala, principalmente se considerarmos que as evidências de validade devem ser interpretadas como um processo cumulativo de investigação. Nesse sentido, as evidências de validade baseadas em critério externo, no caso do fenômeno aqui estudado, mostraram-se um dos estudos mais importantes, dada a necessidade de que o instrumento fosse capaz de diferenciar os grupos investigados, sendo essa a sua principal finalidade. Os resultados aqui apresentados confirmam a adequabilidade do instrumento para ser utilizado dentro de um processo compreensivo para identificação dos estudantes que apresentam sinais indicativos de $\mathrm{AH} / \mathrm{S}$.

\section{REFERÊNCIAS}

ALEGRÍA, I.; LAY, S.; CALDERÓN, C. C.; CÁRDENAS, M. El proceso de construcción y validación de la Escala de Identificacion de Talento Academico Edita. Estudos Pedagógicos, Valdivia, v. 36, n. 2, p. 25-39, 2010.

ALENCAR, E. M. L. S.; FLEITH, D. S.; CARNEIRO, L. B. Gifted education in Brazil: historical background, current practices and research trends. In: WALLACE, B.; SISK, D. A.; SENIOR, J. (ed.). The SAGE handbook of gifted and talented education. London: Sage Publications, 2018. p. 432-445.

AL-HROUB, A.; KHOURY, S. Definitions and conceptions of giftedness around the world. In: KHOURY, S.; AL-HROUB, A. (ed.). Gifted education in Lebanese schools: integrating theory, research and practice. Cham, Switzerland: Springer, 2018. p. 9-38.

ALMEIDA, L. S.; ARAÚJO, A. M.; SAINZ-GÓMEZ, M.; PRIETO, M. D. Challenges in the identification of giftedness: issues related to psychological assessment. Anales de Psicología, Murcia, v. 32, n. 3, p. 621-627, 2016.

ALMEIDA, L. S.; FLEITH, D. S.; OLIVEIRA, E. P. Sobredotação: respostas educativas. Minho: Associação para o desenvolvimento e investigação em Psicologia da Educação, 2013.

ALMEIDA, L. S.; LOBO, C. C.; ALMEIDA, A. I. S.; ROCHA, R. S.; PISKE, F. H. R. Processos cognitivos e de aprendizagem em crianças sobredotadas: atenção dos pais e professores. In: PISKE, F. H. R.; VESTENA, C. L. B.; STOLTZ, T.; MACHADO, M. J. M.; BARBY, A. A. O. M.; BAHIA, S.; FREITAS, S. P. (ed.). Processos afetivos e cognitivos de superdotados e talentosos. Curitiba: Editora Prismas, 2017. p. 17-42.

ALMEIDA, L. S.; OLIVEIRA, E.; MELO, A. Alunos sobredotados: contributos para a sua identificação e apoio. Braga: Aneis, 2000. 
ALMEIDA, M. A.; CAPELLINI, V. L. M. F. Alunos talentosos: possíveis superdotados não notados. Educação, Porto Alegre, v. 55, n. 1, p. 45-64, jan./abr. 2005.

BRACKEN, B. A.; BROWN, E. F. Behavioral identification and assessment of gifted and talented students. Journal of Psychoeducational Assessment, v. 24, n. 2, p. 112-122, 2006.

BRASIL. Ministério da Educação. Lei n. 9.394, de 20 de dezembro de 1996. Estabelece as diretrizes e bases da educação nacional. Disponível em: http://www.planalto.gov.br/ccivil_03/Leis/L9394.htm. Acesso em: 22 fev. 2019.

BRASIL. Políticas públicas para alta habilidade/superdotação. Brasília, DF, 2010. Disponível em: www. senado.gov.br/web/comissoes/CE/AP/AP20080626_superdotados_Cl\%C3\%A1udiaGriboski.pdf. Acesso em: 22 fev. 2010.

BRASIL. Instituto Nacional de Estudos e Pesquisas Educacionais Anísio Teixeira - Inep. Censo Escolar: Educação Especial. Brasília, DF: MEC, 2018. Disponível em: http://portal.inep.gov.br/web/ guest/resultados-e-resumos. Acesso em: 13 mar. 2019.

CLEVELAND, L. M. Examining the relationship between Gifted Behavior Rating Scores and student academic performance. 2017. Thesis (Doctorate of Education) - College of Education, Concordia University, Portland, 2017. Disponível em: https://digitalcommons.csp.edu/cgi/viewcontent. cgi?article=1067\&context=cup_commons_grad_edd. Acesso em: 19 abr. 2019.

DELOU, C. M. C. O atendimento educacional especializado para alunos com altas habilidades/ superdotação no ensino superior: possibilidades e desafios. In: MOREIRA, L. C.; STOLTZ, T. (org.). Altas habilidades/superdotação, talento, dotação e educação. Curitiba: Juruá, 2012. p. 129-142.

FARIAS, E. S.; WECHSLER, S. M. Desafios na identificação de alunos intelectualmente dotados. In: VIRGOLIM, A. M. R. (org.). Altas habilidades/superdotação, inteligência e criatividade: uma visão multidisciplinar. Campinas, SP: Papirus, 2014. p. 335-350.

FREITAS, M. F. R. L.; SCHELINI, P. W. Escala de identificação de dotação e talento: construção de instrumento e validade de conteúdo. Interação em Psicologia, Curitiba, v. 2, n. 2, p. 114-122, 2018.

FREITAS, M. F. R. L.; SCHELINI, P. W.; PÉREZ, R. Escala de identificação de dotação e talento: estrutura e consistência internas. Psico-USF, Campinas, SP, v. 22, n. 3, p. 473-484, 2017.

GILLIAM, J. E.; CARPENTER, B. O.; CHRISTENSEN, J. R. Gifted and talented evaluation scales. Waco, TX: Prufrock, 1996.

GONÇALVES, S. R. V. O curso de pedagogia no Brasil: da formação específica para a formação generalista. Revista Espaço do Currículo, João Pessoa, v. 10, n. 2, p. 244-258, maio/ago. 2017.

GUENTHER, Z. C. Quem são os alunos dotados? Reconhecer dotação e talento na escola. In: MOREIRA, L. C. (org.). Altas habilidades/superdotação: talento, dotação e educação. Curitiba: Juruá, 2012. p. 63-84.

GUIMARÃES, T. G. Avaliação psicológica de alunos com altas habilidades. In: FLEITH, D. S.; ALENCAR, E. M. L. S. (org.). Desenvolvimento de talentos e altas habilidades: orientação a pais e professores. Porto Alegre: Artmed, 2007. p. 79-86. 
HERTZOG, N. B.; MUN, R. U.; DURUZ, B.; HOLLIDAY, A. A. Identification of strengths and talents in young children. In: PFEIFFER, S. I. (ed.). APA handbook of giftedness and talent. Washington: American Psychological Association, 2018. p. 301-316.

IRBY, B.; LARA-ALECIO, R. Attributes of Hispanic gifted bilingual students as perceived by bilingual educators in Texas. SABE Journal, v. 11, p. 120-143, 1996.

JAROSEWICH, T.; PFEIFFER, S. I.; MORRIS, J. Identifying gifted students using teacher rating scales: a review of existing instruments. Journal of Psychoeducational Assessment, v. 20, p. 322-336, 2002 .

JOHNSON, E.; SEMMELROTH, C. L. Special education teacher evaluation: why it matters, what makes it challenging, and how to address these challenges. Assessment for Effective Intervention, v. 39, n. 2, p. 71-82, mar. 2014.

KAUFMAN, J. M.; ANASTASIOU, D.; MAAG, J. W. Special education on the crossroad: an identity crisis and the need for a scientific reconstruction. Exceptionality, v. 25, n. 2, p. 139-155, 2017.

KAUFMAN, S. B.; STERNBERG, R. J. Conceptions of giftedness. In: PFEIFFER, S. (ed.). Handbook of giftedness in children: psycho-educational theory, research and best practices. New York: Springer, 2008. p. 71-91.

LI, H.; LEE, D.; PFEIFFER, S.; KAMATA, A.; ROSADO, J. Measurement invariance for the Gifted Rating Scales-School Form across five cultural groups. School Psychology Quaterly, v. 24, n. 3, p. 186-198, 2009.

LÓPEZ-RATÓN, M.; RODRÍGUEZ-ÁLVAREZ, M. X. OptimalCutpoints: computing optimal cutpoints in diagnostic tests (Version 1.1-4). 2019. Disponível em: https://CRAN.R-project.org/ package=OptimalCutpoints. Acesso em: 26 fev. 2020.

LÓPEZ-RATÓN, M.; RODRÍGUEZ-ÁLVAREZ, M. X.; CADARSO-SUÁREZ, C.; GUDE-SAMPEDRO, F. OptimalCutpoints: An R package for selecting optimal cutpoints in diagnostic tests. Journal of Statistical Software, v. 61, n. 1, p. 1-36, 2014.

MAIA-PINTO, R. R.; FLEITH, D. S. Percepção de professores sobre alunos superdotados. Estudos de Psicologia, Campinas, SP, v. 19, n. 1, p. 78-90, 2002.

MCCALLUM, R. S.; BRACKEN, B. A. The universal multiple abilities scales examiner's manual. Austin, TX: PRO-ED, 2012.

MCCARNEY, S. B.; ANDERSON, P. D. Gifted evaluation scale. Columbia, MO: Hawthorne Educational Services, 1998.

MENDONÇA, L. D.; RODRIGUES, O. M. P. R.; CAPELLINI, V. L. M. F. Identificação de alunos com sinais de altas habilidades ou superdotação. 2015. Disponível em: https://www.researchgate.net/ publication/285589860_IDENTIFICACAO_DE_ALUNOS_COM_SINAIS_DE_ALTAS_HABILIDADES_ OU_SUPERDOTACAO. Acesso em: ago. 2020.

MIRANDA, L. M.; ARAÚJO, A. M.; ALMEIDA, L. S. Identification of gifted students by teachers: reliability and validity of the cognitive abilities and learning scale. Revista de Investigación y Divulgación em Psicologia y Logopedia, v. 3, n. 2, p. 14-18, 2013. 
NAKANO, T. C.; CAMPOS, C. R. Avaliação psicológica das altas habilidades/superdotação: problemas e desafios. In: CAMPOS, C. R.; NAKANO, T. C. (org.). Avaliação Psicológica direcionada a populações específicas. São Paulo: Vetor, 2019. 2 v. p. 99-128.

NAKANO, T. C.; CAMPOS, C. R.; SANTOS, M. V. Escala de avaliação das altas habilidades/ superdotação - versão professor: validade de conteúdo. Estudos Interdisciplinares em Psicologia, Londrina, PR, v. 7, n. 1, p. 103-123, 2016.

NAKANO, T. C.; GOZZOLI, M. Z.; ALVES, R. J. R.; CAMPOS, C. R. Investigación de la eficácia de uma escala de evaluación de altas habilidades - versión professor. Revista de Estudios y Experiencias en Educación, v. 15, n. 29, p. 83-94, 2016.

NAKANO, T. C.; MILIANI, A. F. M.; CAPOROSSI, D. C.; GOZZOLI, M. Z. Evidências de validade do subteste de criatividade verbal da bateria de avaliação das altas habilidades/superdotação. Revista Iberoamericana de Criatividade e Inovação, Campinas, SP, v. 1, n. 1, p. 13-25, 2020.

NAKANO, T. C.; OLIVEIRA, K. S. Triagem de indicadores de altas habilidades/superdotação: estrutura fatorial. Avaliação Psicológica, Campinas, SP, v. 18, n. 4, p. 448-456, 2019.

OLIVEIRA, A. R. A.; AMORIM, R. M.; PIZZI, L. C. V. Disciplina profissão docente em um curso de Pedagogia: trajetórias, experiências e inovações na formação docente. Revista Eletrônica de Educação, São Carlos, SP, v. 12, n. 1, p. 263-278, 2018.

OZCAN, D.; KAYA, F. What does giftedness mean according to teachers? International Journal of Learning and Teaching, v. 8, n. 2, p. 150-155, 2016.

PEDRO, K. M.; OGEDA, C. M. M.; MORAES, L. A. P.; CHACON, M. C. M. Altas habilidades ou superdotação: levantamento dos artigos indexados no SciELO. Interfaces da Educação, v. 7, n. 19, p. 275-295, 2016.

PÉREZ, S. G. P. B. E que nome daremos à criança? In: MOREIRA, L. C.; STOLTZ, T. (org.). Altas habilidades/superdotação, talento, dotação e educação. Curitiba: Juruá, 2012. p. 45-62.

PFEIFFER, S. I. Identifying gifted and talented students: recurring issues and promising solutions. Journal of Applied School Psychology, v. 1, p. 31-50, 2002.

PFEIFFER, S. I.; BLEI, S. Gifted identification beyond the IQ test: rating scales and other assessment procedures. In: PFEIFFER, S. I. (org.). Handbook of giftedness in children: psycho-educational theory, research and best practices. New York: Springer, 2008. p. 177-198.

PFEIFFER, S. I.; JAROSEWICH, T. Gifted Rating Scales: manual. San Antonio, TX: Pearson, 2003.

PFEIFFER, S. I.; PETSCHER, Y. Identifying young gifted children using the Gifted Rating Scales-Preschool: Kindergarten Form. Gifted Child Quartely, v. 52, n. 1, p. 19-29, 2008.

R CORE TEAM. R: A language and environment for statistical computing [Computer software], 2018. Disponível em: https://cran.r-project.org/. Acesso em: 20 jan. 2020.

RENZULLI, J. S. La educación del sobredotado y el desarrollo del talento para todos. Revista de Psicologia (Lima), v. 26, n. 1, p. 25-44, 2008. 
RENZULLI, J. S.; SMITH, L. H.; WHITE, A. J.; CALLAHAN, C. M.; HARTMAN, R. K.; WESTBERG, K. L. Scales for rating the behavior characteristics of superior students. Revised edition. Manseld Center, CT: Creative Learning Press, 2002.

ROBINSON, A.; CLINKENBEARD, P. R. History of giftedness: perspectives from the past presage modern scholarship. In: PFEIFFER, S. I. (org.). Handbook of giftedness in children: psycho-educational theory, research and best practices. New York: Springer, 2008. p. 13-31.

RYSER, G. R.; MCCONNELL, K. Scales for identifying gifted students. Waco, TX: Prufrock Press, 2004.

SAHIN, F. The scale for rating the behavioral characteristics of gifted and talented students: study of factor structure, reliability and validity. Journal of Educational Sciences, v. 38, p. 119-132, 2013.

SELKER, R.; LOVE, J.; DROPMANN, D. jmv: the “jamovi” analyses (Version 0.9.6.1), 2019. Disponível em: https://CRAN.R-project.org/package=jmv. Acesso em: 20 jan. 2020.

SILVERMAN, L. K. Assessment of giftedness. In: PFEIFFER, S. I. (ed.). Handbook of giftedness in children: psychoeducational theory, research, and best practices. Cham, Switzerland: Springer, 2018. p. 183-208.

SUÁREZ, J. T.; WECHSLER, S. M. Escala de identificação de talentos pelo professor, ITP: evidências de validade e precisão. Revista de Educação Especial, Santa Maria, RS, v. 32, p. 1-21, 2019.

THE JAMOVI PROJECT. jamovi. (Version 0.9) [Computer Software]. 2019. Disponível em: https:/| www.jamovi.org. Acesso em: 20 jan. 2020.

VEIGA, E. C.; GRANDE, D.; GROCHOSKI, S. As relações entre o aluno com altas habilidades/ superdotação e o professor do ensino comum. Psicologia Argumento, Curitiba, v. 31, n. 72, p. 23-34, jan./mar. 2013.

VIRGOLIM, A. M. R.; KONKIEWITZ, E. C. Altas habilidades/superdotação, inteligência e criatividade. Campinas: Papirus, 2014.

WILLIAMS, L. Supporting the development of academic talent: the perspectives of students, parents and teachers. In: BALLAM, N.; MOLTZEN, R. (ed.). Giftedness and talent: Australian perspectives. Singapore: Springer, 2017. p. 277-304.

ZAIA, P.; NAKANO, T. C. Escala de Identificação das Altas Habilidades/Superdotação: evidências de validade de critério. Revista Iberoamericana de Diagnóstico y Evaluación e Avaliação Psicológica, Lisboa, v. 55, p. 31-41, abr./jun. 2020.

ZAIA, P.; NAKANO, T. C.; PEIXOTO, E. M. Escala de Identificação de Características de Altas Habilidades/Superdotação: análise da estrutura interna. Estudos de Psicologia, Campinas, SP, v. 35, n. 1, p. 39-51, 2018.

Recebido em: 3 SETEMBRO 2019

Aprovado para publicação em: 8 MAIO 2020 